\begin{tabular}{|c|c|}
\hline \multirow{3}{*}{ 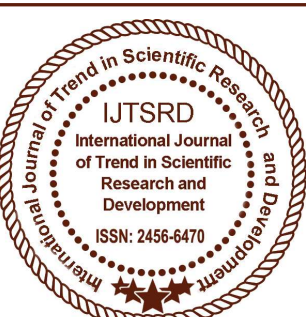 } & $\begin{array}{l}\text { International Journal of Trend in Scientific } \\
\text { Research and Development (IJTSRD) }\end{array}$ \\
\hline & International Open Access Journal \\
\hline & ISSN No: 2456 - 6470 | www.ijtsrd.com | Volume - 2 | Issue - 3 \\
\hline
\end{tabular}

\title{
Secure Routing Packet Transmission With Dynamic Topologies
}

\author{
${ }^{1}$ K. S. N. S Akhila, ${ }^{2}$ K. Raviteja, ${ }^{3}$ M. Sai Balaram Chowdary, ${ }^{4}$ B. Rachana Choudary, ${ }^{5}$ P. V Hari Prasad \\ ${ }^{5}$ Associate Professor \\ $1,2,3,4,5$ Dhanekula Institute of Engineering and Technology, \\ Ganguru, Vijayawada, Andhra Pradesh, India
}

\begin{abstract}
When a text file is transmitting from source node to destination node in the form of packets, sometimes there is a chance of missing last-packet or some packets fail to reach their destination. This problem arises due to the interruption occurred in the network, but these interruptions in the network causes inconvenience to the end-users. In order to overcome this problem, we are implementing a system using Starvation Free Back Pressure (SF-BP) Algorithm which avoids the starvation of packets at the destination and also maximizes the network throughput. This can be achieved by generating the frequencies to each and every node. By using these frequencies, the path will be selected for the data transmission. This ensures that each and every packet is transmitted safe and securely without any interruptions in the network.
\end{abstract}

Keywords: Backpressure, Starvation free, Network throughput

\section{INTRODUCTION}

\section{A. Background}

In the Queue length backpressure routing algorithm during the transmission of packets, there is a chance of losing some packets when any malfunction is generated. Due to this malfunction the packets does not reach their destination. This causes inconvenience to the users' privacy. To avoid this problem, we are using Starvation Free Back Pressure (SFBP) Algorithm which is the extension of the Q-BP algorithm, the traditional two-phase algorithm, in which at first paths are being discovered and only then they are used to send data, may be less effective, as by the time these paths are discovered the topology changes again. This may eventually lead to an accumulation of packets in the network and even to instability, due to infinite looping, poor use of the network resources, and large overhead in maintaining the routes. Instead, Q-BP attempts to exploit congestion gradients in order to achieve maximum network throughput. To do so, it assumes slotted time, and at each time slot, makes routing and resourceallocation decisions based on the maximization of the

sum of the queue-length-differentials multiplied by the corresponding link capacities. It can be proved to achieve stability by showing a negative drift on a quadratic Lyapunov function that represents the sum of squared queue lengths.

\section{B. RELATED WORK}

Many delay-reduction techniques have been introduce. However, while these solutions may reduce the mean delay in the network, they hold no promises regarding individual packet arrivals. Namely, individual packets may still suffer from extremely long delays and even fail to reach their destination. In recent years, it has increasingly appeared that a delaybased backpressure technique would be a natural solution to solve these issues. The idea behind such a technique would be to make routing and resource allocation decisions based on explicit delay information, such as the delay of the Head of-Line (HoL) packet of each queue in the network. For instance, proposed a delay-based technique for singlehop networks and achieved a worst-case delay bound, by discarding a small portion of the packets. 
However, this technique does not apply to multi-hop networks. A delay based scheduling scheme was also proposed.

However, it assumes fixed routes, and thus does not address the natural scenarios of dynamic networks where the topology changes and routes must be adapted to fully utilize the network. Newer works have suggested a delay-based scheduling for multihop dynamic networks. However, their approach does not provide routing, which presents a significant challenge in dynamic topologies We consider dynamic routing and power allocation for a wireless network with time varying channels. The network consists of power constrained nodes which transmit over wireless links with adaptive transmission rates. Packets randomly enter the system at each node and wait in output queues to be transmitted through the network to their destinations. We establish the capacity region of all rate matrices $(\lambda \mathrm{ij})$ that the system can stably support - where $(\lambda$ ij $)$ represents the rate of traffic originating at node $i$ and destined for node $\mathrm{j}$.

A joint routing and power allocation policy is developed which stabilizes the system and provides bounded average delay guarantees whenever the input rates are within this capacity region. Such performance holds for general arrival and channel state processes, even if these processes are unknown to the network controller. We then apply this control algorithm to an ad-hoc wireless network where channel variations are due to user mobility, compare its performance with the Grossglauser-Tse (2001) relay model.

The back-pressure algorithm is a well-known throughput-optimal algorithm. However, its delay performance may be quite poor even when the traffic load is not close to network capacity due to the following two reasons. First, each node has to maintain a separate queue for each commodity in the network, and only one queue is served at a time. Second, the backpressure routing algorithm may route some packets along very long routes. In this paper, we present solutions to address both of the above issues, and hence, improve the delay performance of the backpressure algorithm. One of the suggested solutions also decreases the complexity of the queueing data structures to be maintained at each node.
In this paper, we extend recent results on fair and stable resource allocation in wireless networks to include multicast flows, in particular multi-rate multicast. The solution for multi- rate multicast is based on scheduling virtual (shadow) \& quot;traffic \&quot; that \& quot;moves \& quot; in reverse direction from destinations to sources. This shadow scheduling algorithm can also be used to control delays in wireless networks.

\section{SYSTEM ARCHITECTURE}

In this system, we define a starvation-free stability criterion that ensures a repeated vacuation of all network queues. Then, we introduce SF-BP, the first backpressure routing and resource allocation algorithm that is starvation-free stable. We further present stronger per-queue service guarantees and provide tools to enhance weak streams. We formally prove that our algorithm ensures that all packets reach their destination for wide families of networks.

Finally, we verify our results by extensive simulations using challenging topologies as well as random static and dynamic topologies.

The main advantages of our system are:

Reduce the mean delay in the network; they hold no promises regarding individual packet arrivals. It improves the delay performance in the network.

\section{Main Modules \\ BGP Router:}

The BGP Router is responsible to route the nodes using BGP routing, where the goal is to find a minimal number of relay node locations that can allow shortest-path routing between the sourcedestination pairs, BGP Router consider a one-to-many destination where we want to improve routing between a single source and many destinations. BGP routing table contains valid paths from its source to the entire set of nodes. BGP is also responsible for storing the possible path to destination, can view the recent routing path to destination with their tags Filename, Recent Path, Destination, DIP, Delay and date and time.

\section{EndUsers (Destination)}

In this module, the End user (Node A, Node B, Node $\mathrm{C}$, Node D .............) is responsible to receive the file from the Service Provider In the shortest-path routing 
between the source-destination nodes, the system consists of a one-to-many relationship. Where end User receives file from a single source to destination (Node A, Node B, Node C, Node D.............).

\section{Overlay Router}

The Overlay Router is responsible to route the file to the specified destination, the overlay routing scheme is the set of the shortest physical paths simplifies the execution of this system, finding a minimal path to the destination using overlay routing, one can perform routing via shortest paths, the router is also responsible for Assigning the cost and also can view the cost of nodes with their tags from the node (from), To the node (to) and the cost.

\section{Service Provider}

In this module, the Service Provider calculates the shortest path to Destination, The shortest-path routing over the Internet BGP-based router. The Service provider browses the required file and uploads their data files to the Specified End User (A, B, C, D) and with their DIP (Destination IP) of End User.

The below table displays the list of the shortest path details available from the Source node to the Destination Node through which the packets are transmitting without any packet loss and avoiding the starvation.

\section{CONCLUSION}

The system is implemented by using Starvation Free Back Pressure (SF-BP) Algorithm which avoids the extensive problems: delay and packet loss information.

Based on our simulation performance we find that the stochastic routing is taken to achieve significant results gain in link delay and packet loss negative results in traditional routing. The proposed stochastic routing Algorithm decreases 20\%-40\% delay in the deterministic shortest path and routing is minimal hop routing. This algorithm is also integrated with Forward ErrorCorrection (FEC) for achieving better modelling delivery for reducing nearly $50 \%$ packet loss effect than two different algorithms. The stochastic routing uses the statistical properties of overlay links. So by implementing this system we are developing an efficient way of packet transmission by providing frequencies to the nodes using Starvation Free Back Pressure (SF-BP) algorithm which ensures to provide safe and secure packet transmission using dynamic topologies without any packet loss and avoiding delay in packet transmission.

\section{REFERENCES}

1. R. Albert and A.-L. Barabási, "Statistical mechanics of complex net-works," Rev. Mod. Phys., vol. 74, no. 1, pp. 47-51, Jan. 2002.

2. M. Alresaini, K.-L. Wright, B. Krishnamachari, and M. J. Neely, "Backpressure delay enhancement for encounter based mobile networks while sustaining throughput optimality," IEEE/ACM Trans. Netw, vol. 24,no. 2, pp. 11961208, Apr. 2016.

3. L. Bui, R. Srikant, and A. Stolyar, "Novel architectures and algorithms for delay reduction in back-pressure scheduling and routing," in Proc. IEEE INFOCOM, Apr. 2009, pp. 2936-2940.

4. Y. Cui, E. M. Yeh, and R. Liu, "Enhancing the delay performance of dynamic backpressure algorithms," IEEE/ACM Trans. Netw., vol. 24,no. 2, pp. 954-967, Apr. 2016.

5. A. Dimakis and J. Walrand, "Sufficient conditions for stability oflongest-queue-first scheduling: Second-order properties using fluid lim-its," Adv. Appl. Probab., vol. 38, no. 2, pp. 505-521, 2006.

6. A. Eryilmaz and R. Srikant, "Fair resource allocation in wireless networks using queuelength-based scheduling and congestion control,"in Proc. IEEE INFOCOM, vol. 3. Apr. 2005, pp. 1794-1803.

7. L. Georgiadis, M. J. Neely, and L. Tassiulas. Resource Allocation and Cross-Layer Control in Wireless Networks. Breda, The Netherlands: Now 2006 